\title{
Eye Position Signal Modulates a Human Parietal Pointing Region during Memory-Guided Movements
}

\author{
Joseph F. X. DeSouza, ${ }^{1}$ Sean P. Dukelow, ${ }^{1}$ Joseph S. Gati, ${ }^{3}$ Ravi S. Menon, ${ }^{3}$ Richard A. Andersen, ${ }^{4}$ and \\ Tutis Vilis ${ }^{2}$ \\ ${ }^{1}$ Graduate Program in Neuroscience, Siebens-Drake Research Institute and 2 Department of Physiology and \\ Ophthalmology, University of Western Ontario, London, Ontario, Canada N6G 2V4, ${ }^{3}$ Advanced Imaging Labs, The John P. \\ Robarts Research Institute, London, Ontario, Canada N6A 5K8, and ${ }^{4}$ Division of Biology, California Institute of Technology, \\ Pasadena, California 91125
}

\begin{abstract}
Using functional magnetic resonance imaging, we examined the signal in parietal regions that were selectively activated during delayed pointing to flashed visual targets and determined whether this signal was dependent on the fixation position of the eyes. Delayed pointing activated a bilateral parietal area in the intraparietal sulcus (rIPS), rostral/anterior to areas activated by saccades. During right-hand pointing to centrally located targets, the left rIPS region showed a significant increase in activation when the eye position was rightward compared with leftward. As expected, activation in motor cortex showed no modulation when only eye position changed. During pointing to retinotopically identical targets, the left rIPS region again showed a significant increased signal when the eye position was rightward
\end{abstract}

compared with leftward. Conversely, when pointing with the left arm, the right rIPS showed an increase in signal when eye position was leftward compared with rightward. The results suggest that the human parietal hand/arm movement region (rIPS), like monkey parietal areas (Andersen et al., 1985), exhibits an eye position modulation of its activity; modulation that may be used to transform the coordinates of the retinotopically coded target position into a motor error command appropriate for the wrist.

Key words: eye position; extraretinal signal; position modulation; spatial transformation; pointing; memory-guided; parietal cortex; intraparietal sulcus; IPS; functional magnetic resonance imaging; fMRI; human
Pointing and reaching to objects of interest involves complex visual sensory-to-motor transformations thought to be computed in the parietal cortex (Milner and Goodale, 1995). Neurons active for hand and arm movements have been recorded from a multitude of areas along the monkey intraparietal sulcus, including parietal reach region (PRR) and anterior intraparietal (AIP) (Mountcastle et al., 1975; Sakata et al., 1995, 1997; Snyder et al., 1997). Neurons in PRR, which includes areas medial intraparietal and parietooccipital, were found to become active during reaching movements (Snyder et al., 1997) (for review, see Colby and Goldberg, 1999). Neurons within AIP have been shown to be active in the monkey during movements of the hand, as well as grasping (Gallese et al., 1994; Murata et al., 1996). In positron emission topography imaging of humans, Kawashima et al. (1996) showed that reachingrelated areas were more anterior along the intraparietal sulcus than saccade-related areas. Using functional magnetic resonance imaging (fMRI), Binkofski et al. (1998) found bilateral activation of the lateral bank of the anterior intraparietal sulcus (IPS) for fine finger movements during grasping of an object compared with pointing to the object. They suggested a distinct cortical area in the anterior portion of the human intraparietal sulcus controls precisely tuned finger movements.

To direct the hand-arm to objects in space on the basis of the image seen by the retina requires several coordinate transformations. One key transformation is to convert the image from eye-

Received Nov. 18, 1999; revised May 15, 2000; accepted May 15, 2000.

This research was supported by the Medical Research Council of Canada and Human Frontiers in Science. J.F.X.D. was supported by National Sciences and Engineering Council of Canada and Medical Research Council of Canada. We thank L. Van Cleeff and Jason Connolly for assistance during data collection, L. Van Cleeff for the design and construction of equipment, and Jody Culham and Douglas Tweed for invaluable feedback.

Correspondence should be addressed to Dr. Tutis Vilis, The MRC Group for Action and Perception, Department of Physiology, Medical Sciences Building, University of Western Ontario, London, Ontario, Canada N6A 5C1. E-mail: Tutis.Vilis@med.uwo.ca.

Copyright (C) 2000 Society for Neuroscience $0270-6474 / 00 / 205835-06 \$ 15.00 / 0$ centered coordinates into a target location with respect to the head by taking into account the position of the eyes in the orbit (Andersen et al., 1993). One tool used for this sensory-to-motor coordinate transformation is gain field modulation (Andersen et al., 1985) of neurons in the parietal cortex. That is, neurons in the inferior parietal cortex in primates change their visual responsiveness to retinotopically identical stimuli with changes in eye position (Andersen et al., 1985, 1990). Gain modulation of neural activity by eye, head, vestibular, and body position signals has been subsequently shown in cortical areas from visual cortex through to premotor cortex (Brotchie et al., 1995; Galletti et al., 1995; Ferraina et al., 1997; Mushiake et al., 1997; Andersen et al., 1998; Boussaoud et al., 1998; Snyder et al., 1998; Boussaoud and Bremmer, 1999; Bremmer et al., 1999; Nakamura et al., 1999; Trotter and Celebrini, 1999).

We collected fMRI images from human parietal cortex while subjects pointed their hand to flashed visual targets. Pointingrelated activity was found along the intraparietal sulcus. A subregion that was selectively activated during only pointing was located anterior to regions also involved in saccades. In this subregion, rostral IPS (rIPS), pointing-related activity was modulated by eye position in the orbit, suggesting that this region may be involved in transforming the retinotopic visual signal to a head- or bodycentered representation.

Parts of this paper have been published previously (DeSouza et al., 1998)

\section{MATERIALS AND METHODS}

Subjects and imaging sessions

Eight healthy adults (five males, three females; mean \pm SD, $26.9 \pm 2$ years of age) with no known behavioral or neuroanatomical anomalies were paid volunteers in this study. Six subjects were right-handed and two were left-handed as determined by a modified Edinburgh inventory (Oldfield, 1971). All subjects provided informed written consent, and the University of Western Ontario Ethics Review Board approved the study. Each subject was well trained to make delayed pointing and delayed saccade movements to visual stimuli, having practiced $1 \mathrm{~d}$ before, just before entering the magnet and in the magnet bore just before imaging. Four subjects each 


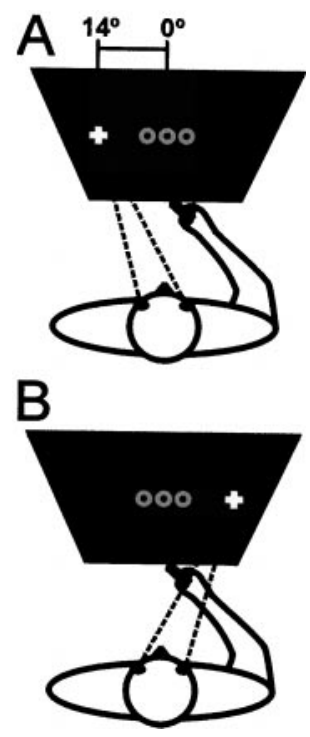

Figure 1. A, Schematic view of the subject laying in the magnet bore while pointing to one of three flashed target positions (represented by the gray circles at the center) and fixating the left cross or performing the same pointing movements while fixating on the right cross $(B)$.

underwent four separate imaging sessions, three subjects each underwent three imaging sessions, and the remaining subject underwent one session for a total of 26 imaging sessions.

\section{Apparatus for pointing and saccades within the MRI}

A pointing task with a direct view of the visual targets was designed that complied with the constraints of the MRI. Subjects lay supine and looked directly at and/or pointed to targets projected onto a screen taped to the top of the magnet bore. The computer-generated visual targets were projected from a liquid crystal display projector (NEC MT800) through a series of camera lenses and reflected by a mirror onto the bore screen. The bore was dark except for the projection of the visual stimulus. During pointing, the subject's view of their hand was occluded with black cardboard.

To stabilize the head during the pointing movements, vacuum beanbag pillows (Olympic Medical, Seattle, WA.) surrounded the head, neck, shoulders, and under the pointing arm. Straps were placed across the torso and upper arm to prevent arm motion from translating to the head. A dental impression bite bar was also used.

\section{Visual stimulus and experimental paradigms}

Stimuli for pointing tasks. The sequence of events for the delayed pointing task was as follows: (1) fixation was maintained on a cross $14^{\circ}$ to the left (Fig. $1 A$ ) or right of center (Fig. $1 B$ ); (2) a target for pointing, consisting of a gray cross on a black background, was flashed $(100 \mathrm{msec})$ at one of three locations $\left(4^{\circ}\right.$ left, center, or $4^{\circ}$ right) (Fig. 1, three circle positions); and (3) after a short delay $(750-1250 \mathrm{msec})$, the subject was instructed to initiate a pointing movement by a brief disappearance of the fixation cross. Subjects touched the remembered target position in near darkness while maintaining eccentric visual fixation on the fixation cross.

The pointing movement consisted of rotating the wrist (partially aided by elbow extension) to lightly touch the screen with the index finger at the remembered target position. All subjects were instructed to point using a preshaped hand position such that the index finger was pointing and the remaining fingers were tucked under the palm. Subjects were also instructed to make the movement as naturally as possible within the constraints of the magnet bore. The distance for a pointing movement from resting position (on the subjects' chest) to the screen ranged from 22 to 29 $\mathrm{cm}$. Equal numbers of delayed pointing movements (eight) were made during each block with an intertrial interval between 1.0 and $1.3 \mathrm{sec}$.

Four experiments were conducted on separate days each using a block design. In experiment Ia, the fixation position of the eye changed from block to block while the pointing targets remained the same. Within a block, subjects $(n=7)$ fixated $14^{\circ}$ left (Fig. $\left.1 A\right)$ or $14^{\circ}$ right (Fig. $\left.1 B\right)$ and pointed to one of three remembered positions located at center or at $\pm 4^{\circ}$ Thus, within a block, there was no change in fixation target. In the control task, visual fixation was at a center, with no visual targets flashed, and the hand-arm was at a resting position.

In experiment $\mathrm{Ib}$, the eccentricity of the fixation of the eye was varied from experiment Ia. Subjects $(n=6)$ fixated at \pm 6 or $\pm 20^{\circ}$ and pointed to one of three remembered positions located at center or at $\pm 2^{\circ}$. For this and the remaining experiments, the control task used exactly the same visual stimuli, but subjects did not point. Subjects were cued by the color of the fixation cross (red, to fixate without any arm movement; green, to fixate and point).

In experiment IIa, the retinotopic location of the visual targets for pointing was kept constant while both the fixation location and the pointing location was changed. Subjects $(n=7$ using right hand and, in a different imaging session, $n=6$ using left hand) fixated at $\pm 20^{\circ}$ and pointed to one of three remembered positions $\pm 2^{\circ}$ apart and centered $\pm 6^{\circ}$ to the left or right of the fixation crosses (see Fig. $4 A$ ).

In experiment IIb, the fixation position of the eye was kept constant while the pointing target was changed. Here the fixation cross was always at $20^{\circ}$ to the left while targets for delayed pointing were centered $6^{\circ}$ to the right and $46^{\circ}$ to the right of fixation (see Fig. $4 A$, points 1,2$)(n=7$ subjects using the right hand, and in a different imaging session, $n=6$ using the left hand).

Stimuli for saccade tasks. We wished to limit our region of interest (ROI) to those involved in pointing and to exclude those involved in generating saccades. To identify saccade-related voxels, a separate experiment was performed subsequent to the pointing experiments. Subjects were instructed to make horizontal saccades ( 4 or $8^{\circ}$ amplitude) to a flashed gray cross $\left(2^{\circ}\right.$ large $)$ displayed on a black background. During a block, there were three possible saccade target positions (all $4^{\circ}$ apart), which were centered either $14^{\circ}$ to the left or right. Targets were briefly $(50 \mathrm{msec})$ flashed, and then after a random delay of 750-1000 msec the fixated cross would disappear instructing the subject to make a saccade to the remembered spatial location. There were no visual targets on the screen when a delayed saccade was executed. Equal numbers of delayed saccades (13) were made during each block with an intertrial interval of $0.75-1.4 \mathrm{sec}$ (for comparison, the pointing experiments described previously involved eight movements).

Functional scanning. Each subject completed six to eight $7.5 \mathrm{~min}$ functional scans and an anatomical scan during each imaging session. A functional scan consisted of four repetitions of an $A_{1}-B-A_{2}-B$ block design with the $\mathrm{A}$ blocks being the motor tasks (pointing or saccades depending on the experiment) and the B block being the visual fixation controls. Each block was 27-28 sec in length (a 4-10 sec fixation period occurred at the beginning of each scan). Each subject completed four to six repetitions of the pointing experiment and two repetitions of the saccade experiment in each imaging session.

\section{MRI parameters}

Experiments were performed on a 4.0-Tesla Varian Siemens (Palo Alto, CA and Erlangen, Germany) UNITY INOVA whole-body imaging system equipped with whole-body shielded gradients. A cylindrical quadrature birdcage radio frequency (RF) coil was used for transmission and reception of RF signal (Barberi et al. 2000). Eleven contiguous slices were used to image the entire parietal cortex, with some occipital and frontal cortex within the imaged planes. For experiment Ia, functional data were collected using navigator echo corrected $\mathrm{T} 2 *$-weighted segmented gradient echoplanar imaging [11 slices, $64 \times 64$ resolution, $20 \mathrm{~cm}$ in plane field of view (FOV), time to echo (TE) of $15 \mathrm{msec}$, volume acquisition time of 1.5 sec, and a voxel size of $3.1 \times 3.1 \times 6 \mathrm{~mm}]$.

For all other experiments, the functional echoplanar images were centered about the previously imaged region of the intraparietal sulcus region (because subjects had this region mapped from experiment Ia). Imaging parameters were the same as experiment Ia except for a $19.2 \mathrm{~cm}$ FOV, 3 $\mathrm{mm}$ slice thickness, and an in-plane resolution of $3 \times 3 \mathrm{~mm}$. Volume acquisition time was $2 \mathrm{sec}$. Functional data were superimposed on highresolution inversion prepared three-dimensional T1-weighted anatomical images of the brain ( 64 slices, $256 \times 256$, TE of $6.2 \mathrm{msec}$, time to relaxation of $11.4 \mathrm{msec}$ ) using a phase reference image that corrected for high-field geometric distortions.

\section{Image analysis}

We excluded any scans (four) in which motion artifacts were observed in a cinematic loop. Time courses within each voxel were corrected for linear drift. Anatomical and functional images were transformed to the coordinate system of Talairach and Tournoux (1988) by using a spatial normalization template. A simple piecewise linear resampling algorithm was used. The analysis used a dependent $t$ test to compare motor states (pointing or saccade) with the fixation states $(p<0.001)$ using the imaging software Stimulate 5.1 (Strupp, 1996). Multiple scans of the same experiment were averaged to increase voxel signal-to-noise. The pointing scans from each imaging session were used to map pointing voxels. Forman et al. (1995) showed that voxels that pass a cluster-size threshold of greater than three have increased confidence thresholds from a $t$ test value of $p<0.005$ to a corrected probability of less than $p<0.00001$. A cluster-size threshold of greater than three was used after voxels passed the dependent $t$ test. Hence, our corrected $p$ value would be $<0.00001$.

Our selected ROI was limited to voxels activated only by pointing. First, we identified the voxels involved in pointing and then excluded those voxels that were also involved in saccades. The pointing voxels were defined by the subtraction of activation during pointing minus that during fixation, independent of where eye position was. Care was taken to ensure that the functionally activated region was within the cortex lining the rostral/ anterior intraparietal sulcus region and not within the postcentral gyrus using the sulcal anatomy from the high-resolution anatomical images. 


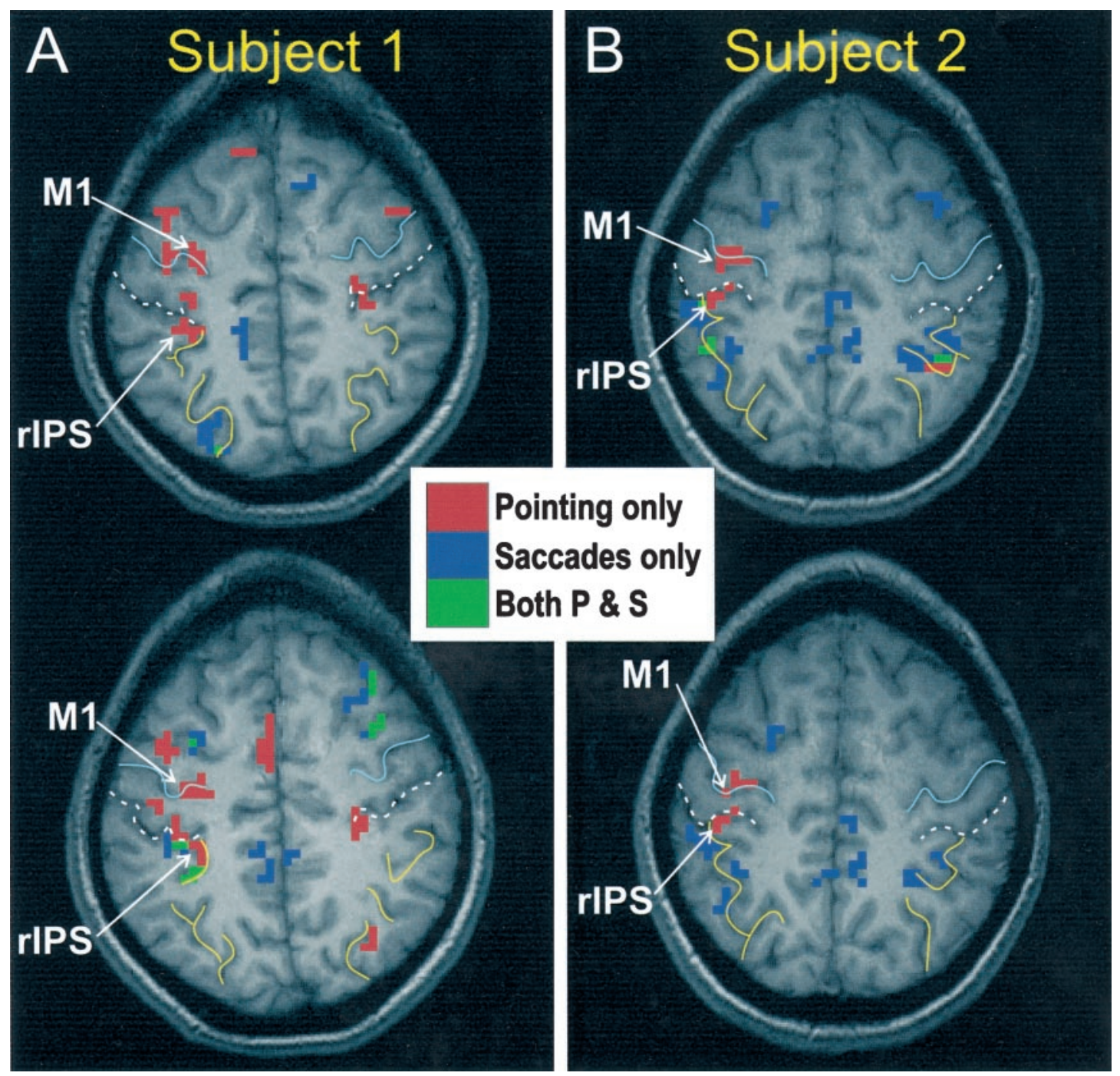

Figure 2. A, Two axial-oblique anatomical slices showing functional activation along the intraparietal sulcus region from a representative subject. The slices show voxels activated $(p<0.0001)$ during pointing only $($ red $)$, during saccades only (blue), and during both pointing and saccades $($ green $)$. B, A second subject. The thin light blue line outlines the central sulcus, the dotted white line outlines the postcentral sulcus, and the yellow lines outline the various branches of the intraparietal sulcus in each subject. M1, Motor cortex activation. Left in each image is the left hemisphere.

Finally, the signal intensity from these rIPS pointing-only voxels was examined for an eye position effect. The same analysis was conducted for other brain regions activated for pointing only, including the motor cortex

\section{RESULTS}

\section{Experiment I: changing eye position while pointing at identical targets}

In experiment Ia, we examined the effect of eye position on delayed pointing by comparing parietal pointing-related activity while subjects fixated at $14^{\circ}$ to the left and $14^{\circ}$ to the right of center (Fig. $1 A, B)$. In all subjects, voxels activated by pointing with the right hand (Fig. $2 A, B$, red voxels) were located along the left rIPS, rostral/anterior to saccade-related voxels (green and blue voxels). The rIPS activation was consistently rostral/anterior with respect to the saccade activation along the cortex lining the intraparietal sulcus. These functionally pointing-only activated voxels showed, on average, a significant modulation by eye position $\left(t_{(6)}=3.05\right.$, $p<0.05$ ) (Figure $3 A$ ). As would be expected given that subjects pointed to the same targets, the left motor cortex, located anterior to the central sulcus, showed no eye position modulation $\left(t_{(6)}=\right.$ 1.28, $p<0.25$ ) (Fig. $3 A$, far right bars). This pointing task also activated other areas, the postcentral cortex, the premotor cortex, supplementary motor cortex, dorsolateral prefrontal cortex, MT/
MST (motion complex), and visual areas (V1/V2/V3a), none of which showed a significant modulation by eye position.

To determine whether the rIPS modulation was dependent on the eccentricity of the eye, in experiment Ib, we compared both smaller $\left( \pm 6^{\circ}\right)$ and larger $\left( \pm 20^{\circ}\right)$ fixation eccentricities during pointing to the same central target positions. Surprisingly, whereas there was a significant position modulation of the signal intensity of rIPS voxels when fixation position changed from $6^{\circ}$ left to $6^{\circ}$ right $\left(t_{(6)}=\right.$ $2.94, p<0.05$ ) (Figure 3B), no significant position modulation was observed for the $\pm 20^{\circ}$ change in eye position (Fig. $3 B$, far right bars) across the subjects. This may be attributable to the stimulus being in the far ipsilateral visual field for rightward gaze, which may produce a reduced response (see below) and may counterbalance the eye position effect. Again, voxels along the motor cortex showed no modulation as a function of eye position during pointing.

\section{Experiment II: a change in eye position while keeping targets retinotopically identical}

In the previous series of experiments, the pointing targets were kept the same (always near the midline), and the effect of changing eye fixation eccentricity was examined. Here we examined the effect of changing the position of eye fixation in the orbit while keeping the location of the pointing target retinotopically identical. 

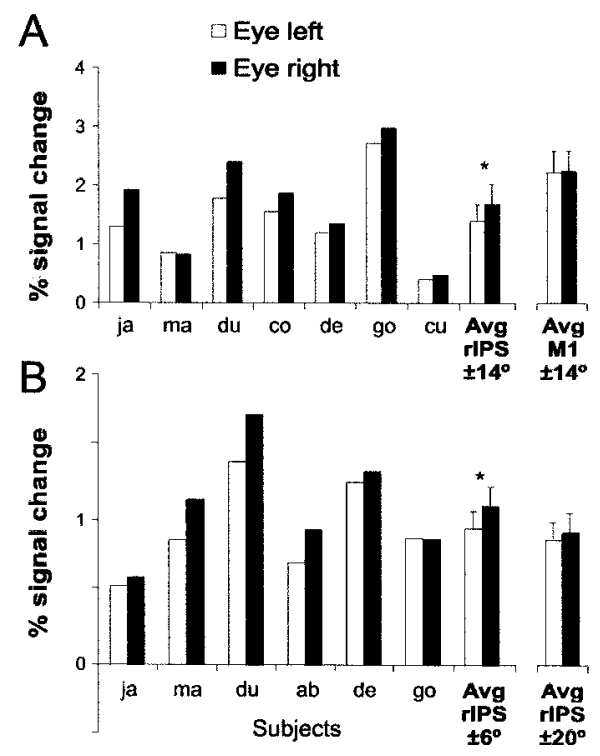

Figure 3. The activation produced by pointing movements of the right hand is modulated by eye position. White bars represent percent signal change during pointing while the eyes were fixating to the left and the black bars while fixating to the right. $A$, The signal activity from left rIPS when fixating $14^{\circ}$ to the left and right in seven subjects, their average and SE ( $A v g$ $\left.r I P S \pm 14^{\circ}\right)$. The far right bars plot the average $(n=7)$ and SE from motor cortex $\left(A v g M 1 \pm 14^{\circ}\right)$. B , The signal intensity from left rIPS as the subjects change eye position from $6^{\circ}$ to the left and the right. The average and SE for the $\pm 6^{\circ}\left(\right.$ Avg rIPS $\left.\pm 6^{\circ}\right)$ and also $\pm 20^{\circ}\left(\right.$ Avg rIPS $\left.\pm 20^{\circ}\right)$ change in eye position is plotted to the right. ${ }^{*} p<0.05$. M1, Motor cortex activation.

To do this, one must of course change the location of the pointing target with respect to the body. In this experiment, fixation was changed from $20^{\circ}$ left to $20^{\circ}$ right while pointing to target positions $\pm 6^{\circ}$ away from fixation. Figure $4 B$ shows the signal time course from left rIPS voxels that was activated during right-hand pointing and not during saccades from a representative subject. Eight pointing blocks (light gray or dark gray shaded regions) are shown alternating with fixation blocks (white regions). For the pointing blocks, eye position was alternated between $20^{\circ}$ to the left (light gray shading) and $20^{\circ}$ to the right (dark gray shading). Targets were in the left visual field for blocks 3 and 4 and in the right visual field for blocks 1 and 2 (Fig. $4 A$, pointing target areas are represented by white ovals with numbers). The four blocks were repeated for a total of eight blocks. When pointing to retinotopically identical targets, the signal was greater when eye position was right (dark gray bars) compared with left (light gray bars) $\left(t_{(13)}=6.38, p<0.0001\right)$. All seven subjects showed a similar eye position modulation (average, $t_{(6)}=5.59, p<0.005$ ) (Fig. $5 A$ ). In separate imaging sessions, the activation during pointing with the left hand was examined. In this case, the signal intensity from right hemisphere rIPS showed an eye position modulation, but the direction of modulation was opposite in sign. Figure $4 C$ shows that there was a signal increase when eye position was left (light gray blocks) compared with right for one subject (dark gray blocks) $\left(t_{(13)}=-6.43, p<0.0001\right)$. A similar modulation was observed in five of the six subjects (average, $t_{(5)}=$ 2.62, $p<0.05$ ) (Fig. $5 C$ ). In contrast, the right motor cortex voxels did not show a statistically significant increase in signal across the population $\left(t_{(5)}=1.33, p<0.24\right)($ Fig. $5 D)$.

Activation was also observed in the ipsilateral-to-hand rIPS in 21 of the 26 imaging sessions ( 15 of 20 for the right hand, and six of six for the left hand). On average, this was not modulated by eye position. The average Talairach coordinates across experiments for rIPS in the left hemisphere were $x=-38 \pm 3, y=-49 \pm 3$, and $z=44 \pm 1 \mathrm{~mm}$, and the right hemisphere were $x=34 \pm 3, y=$ $-46 \pm 3, z=41 \pm 2 \mathrm{~mm}$. The left rIPS activation was posterior and superior to the unilateral activity in the left somatosensory-postcentral cortex $(x=-38 \pm 3, y=-39 \pm 4$, and $z=49 \pm 2)$ (Talairach and Tournoux, 1988).

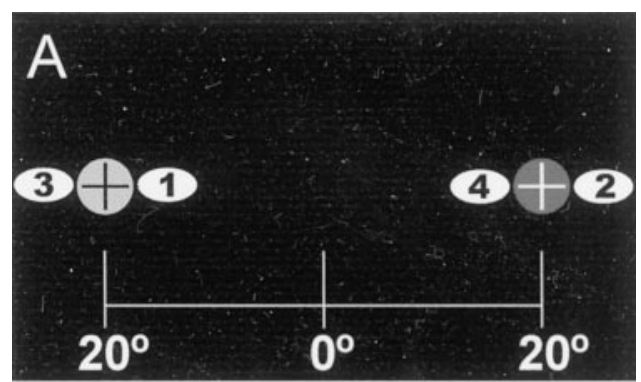

\section{B RIGHT HAND POINTING}

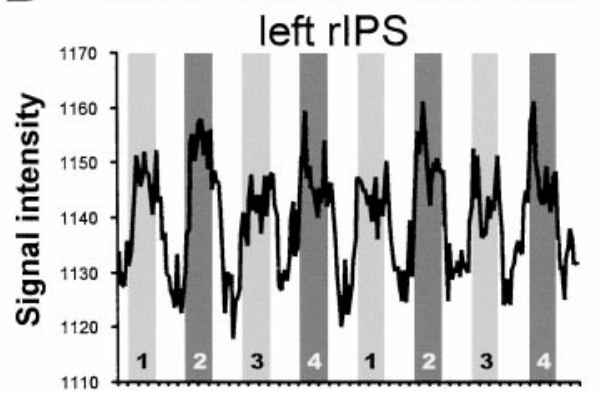

\section{LEFT HAND POINTING}

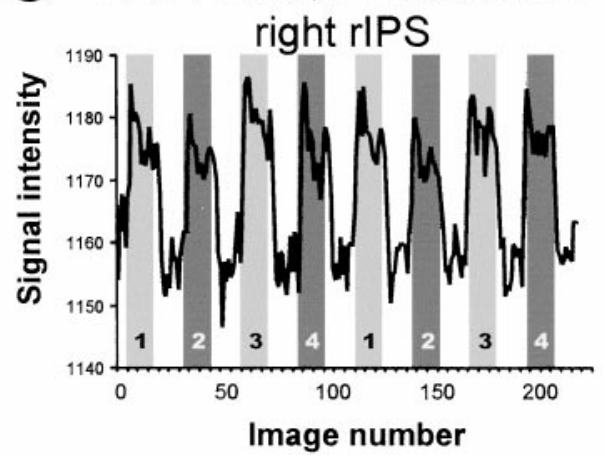

Figure 4. A, Target locations for eye fixation (black cross surrounded by light or dark gray circles) and pointing positions (white ovals with numbers). The numbers within the ovals represent the order of the pointing blocks. $B$, The graph plots signal intensity versus image number (1 image is $2 \mathrm{sec}$ ) from a representative subject's left rIPS voxels during right-hand pointing. The shading of each bar represent fixation position (light gray to the left, dark gray to the right). The numbers correspond to the pointing positions indicated in $A$. The white portions represent the control periods when the subject was fixating but not pointing to the flashed targets. $C$, Same as $B$ but now for the right rIPS during left-hand pointing.

Unlike V1, which is activated only by contralateral visual stimuli, our subjects' rIPS was active during pointing movements to targets flashed in either visual field. Similar to V1, our subjects' left rIPS showed a signal increase for pointing to contralateral versus ipsilateral visual stimuli during right-hand pointing (see Fig. $4 A$, blocks 1 greater than 3 when fixating left, $t_{(6)}=2.55, p<0.05$, and blocks 2 greater than 4 when fixating right, $t_{(6)}=3.50, p<0.05$ ). Also, the right rIPS showed a signal increase for pointing to contralateral versus ipsilateral visual stimuli during left-hand pointing but only when fixating left (blocks 3 greater than $1, t_{(5)}=2.97$, $p<0.05)$.

A lesser but statistically significant modulation was also observed from voxels of the left motor cortex during right-hand pointing $\left(t_{6}\right.$ $=2.86, p<0.05$ ) (Fig. $5 B$ ) (but not in the right motor cortex with left-hand pointing). However, here both the eye fixation position and the position of the pointing targets (and thus hand position) changed simultaneously. To determine whether this modulation in motor cortex was attributable to the change in hand position, in experiment IIb, subjects were required to maintain a constant eye 

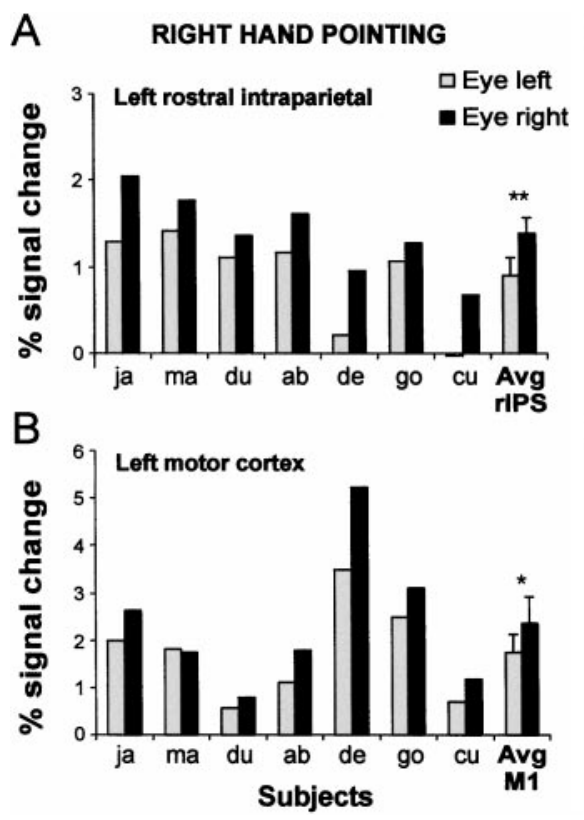

C
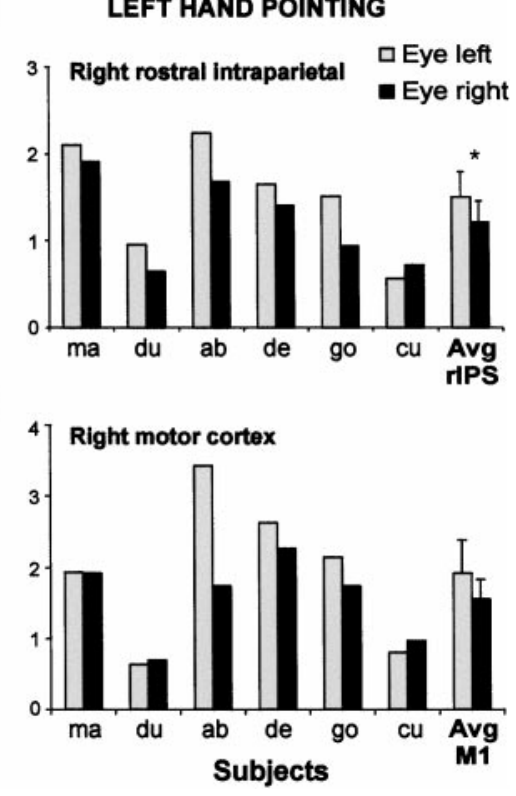

Figure 5. A, The percent signal change from voxels in the left rIPS during pointing with the right hand to retinotopically identical targets while fixating $20^{\circ}$ to the left or right. The individual data of seven subjects as well as their average (Avg rIPS) and SE are plotted. $B$, The same for the left motor cortex. $C$, The same as $A$ except for right rIPS while pointing with the left hand $(n=6) . D$, The same as $C$ except for right motor cortex. ${ }^{*} p<0.05,{ }^{* *} p<0.005$. fixation position while pointing to different spatial positions (Fig. $4 A$, left fixation and pointing positions 1,2 ). Subjects again used the right arm for pointing in one session and the left arm in another session. We found no position modulation in the signal intensity from pointing-only voxels within the rIPS and, surprisingly, no modulation in motor cortex either.

\section{DISCUSSION}

A key step in transforming a target location as seen by the eye into a motor error command appropriate for the wrist is to make the motor signal invariant to changes in eye position. Surprisingly, primate studies have shown that one step in this process involves a modulation of neural activity by changes in the eye position signals (Andersen et al., 1985; Zipser and Andersen, 1988; Boussaoud and Bremmer, 1999). Within the monkey parietal cortex, the intraparietal sulcus appears to be involved in transforming the sensory information to a variety of coordinate frames referenced to the head, arm, body, or world (Andersen et al., 1993, 1998). To achieve this, gain field modulation by different position signals appears to be used, including the position of head (Brotchie et al., 1995), arm position (Andersen et al., 1998), and vestibular signals (Snyder et al., 1998). In humans, the intraparietal sulcus appears to also contain regions that are homologs to those of monkey (Grafton et al., 1996; Kawashima et al., 1996; Kertzman et al., 1997; Binkofski et al., 1999; Baker et al., 1999). Humans with parietal cortex damage show many spatial deficits, such as neglect and extinction (Driver and Mattingley, 1998; Vallar, 1998).

As did Kawashima et al. (1996), we found a pointing area that was more anterior, along the intraparietal sulcus, to voxels whose activity was related to saccades. This subregion, which we call rIPS, exhibited pointing-related activity that was modulated by eye position in the orbit. The direction of the modulation was contraversive (larger for an eye position away from the measured parietal hemisphere) in all of our experiments. This direction was the same as that reported in single-cell recordings in monkeys; activity increases more often than not for the contralateral eye and head positions in areas lateral intraparietal and 7a (Brotchie et al., 1995).

In human fMRI, Baker et al. (1999) also recently showed a contraversive eye position modulation during a nonvisual fingertapping task in motor, premotor, lateral superior, and inferior parietal cortices. This latter area appears to be analogous to our rIPS region. However, unlike Baker et al. (1999) who showed an expansion effect (i.e., more voxels when changing gaze position from left to right), our study showed true gain effects (i.e., the same group of voxels showed increased signal that depended on eye position). This true gain effect was determined using a subtraction that was independent of eye position (pointing minus fixation). The difference between our results and those of Baker et al. (1999) may be attributable to two factors. First, the two tasks differed, directing movement to a visually encoded remembered target in space versus finger tapping that is presumably directed to a proprioceptively encoded location. Second was the selection of voxels. We selected voxels only active in pointing (i.e., excluding voxels with saccaderelated activation). If one considers all pointing-related voxels in rIPS, the true gain effect appears to be diluted. Together, the two results suggest that eye position influences visual sensory-to-motor transformations for finger and hand movements, as well as those that are not visually driven (Baker et al., 1999).

Our evidence, which it is indeed eye position that is producing the modulation in rIPS, is as follows. Experiment I showed that, for the same pointing movement, activity was greater when eye fixation was contralateral. In this experiment, not only did eye position change but so did the visual field in which the target was flashed. Thus, this result could also be interpreted as an increase in activity when the target was in the ipsilateral visual field. To sort this out, in experiment IIa, the target location in the visual field was kept constant while changing both the fixation position and the pointing position. Again, a clear modulation was observed. Finally, no modulation was observed in experiment IIb when only the target pointing position was changed. Thus, this human parietal pointing region may be using eye position signals to transform the visual signals into ones that the premotor and motor cortex could use to execute the appropriate action.

This rIPS region had several characteristics that were intermediate between the visual field-specific characteristics of primary visual cortex and the limb-specific characteristics of primary motor cortex. First, activation in the rIPS region was bilateral in $75 \%$ of the imaging sessions when pointing with the right hand and in all sessions when using the left hand. Second, activation was observed in the rIPS region when pointing to remembered targets in either visual field. Interestingly, when eye position is kept constant, this activation showed a small but significant increase when the target was in the contralateral visual field. These observations are suggestive of a representation within rIPS that is in the process of being transformed by position signals from the eye.

\section{REFERENCES}

Andersen RA, Essick GK, Seigal RM (1985) Encoding of spatial location by posterior parietal neurons. Science 230:456-458.

Andersen RA, Bracewell RM, Barash S, Gnadt JW, Fogassi L (1990) Eye 
position effects on visual, memory, and saccade-related activity in areas LIP and 7a of macaque. J Neurosci 10:1176-1196.

Andersen RA, Snyder LH, Li CS, Stricanne B (1993) Coordinate transformations in the representation of spatial information. Curr Opin Neurobiol 3:171-176.

Andersen RA, Snyder LH, Batista AP, Buneo CA, Cohen YE (1998) Posterior parietal areas specialized for eye movements (LIP) and reach (PRR) using a common coordinate frame. Novartis Found Symp 218:109-122.

Baker JT, Donoghue JP, Sanes JN (1999) Gaze direction modulates finger movement activation patterns in human cerebral cortex. J Neurosci 19:10044-10052.

Barberi EA, Gati JS, Rutt BK, Menon RS (2000) A transmit-only/receiveonly (TORO) RF system for high-field MRI/MRS applications. Magn Reson Med 43:284-289.

Binkofski F, Dohle C, Posse S, Stephan KM, Hefter H, Seitz RJ, Freund HJ (1998) Human anterior intraparietal area subserves prehension: a combined lesion and functional MRI activation study. Neurology 50:1253-1259.

Binkofski F, Buccino G, Stephan KM, Rizzolatti G, Seitz RJ, Freund H (1999) A parieto-premotor network for object manipulation: evidence from neuroimaging. Exp Brain Res 128:210-213.

Boussaoud D, Bremmer F (1999) Gaze effects in the cerebral cortex: reference frames for space coding and action. Exp Brain Res 128:170-180.

Boussaoud D, Jouffrais C, Bremmer F (1998) Eye position effects on the neuronal activity of dorsal premotor cortex in the macaque monkey. J Neurophysiol 80:1132-1150.

Bremmer F, Graf W, Ben Hamed S, Duhamel JR (1999) Eye position encoding in the macaque ventral intraparietal area (VIP). NeuroReport 17:873-878.

Brotchie PR, Andersen RA, Snyder LH, Goodman SJ (1995) Head position signals used by parietal neurons to encode locations of visual stimuli. Nature 375:232-235.

Colby CL, Goldberg ME (1999) Space and attention in parietal cortex. Annu Rev Neurosci 22:319-349.

DeSouza JFX, Dukelow SP, Gati JS, Menon RS, Andersen RA, Vilis T (1998) The human parietal pointing region is modulated by eye position during memory-guided reaching. Soc Neurosci Abstr 24:1145.

Driver J, Mattingley JB (1998) Parietal neglect and visual awareness. Nat Neurosci 1:17-22.

Ferraina S, Johnson PB, Garasto MR, Battaglia-Mayer A, Ercolani L, Bianchi L, Lacquaniti F, Caminiti R (1997) Combination of hand and gaze signals during reaching: activity in parietal area $7 \mathrm{~m}$ of the monkey. J Neurophysiol 77:1034-1038.

Forman SD, Cohen JD, Fitzgerald M, Eddy WF, Mintun MA, Noll DC (1995) Improved assessment of significant activation in functional magnetic resonance imaging (fMRI): use of a cluster-size threshold. Magn Reson Med 33:636-647.

Gallese V, Murata A, Kaseda M, Niki N, Sakata H (1994) Deficit of hand preshaping after muscimol injection in monkey parietal cortex. NeuroReport 5:1525-1529.
Galletti C, Battaglini PP, Fattori P (1995) Eye position influence on the parieto-occipital area PO (V6) of the macaque monkey. Eur J Neurosci 7:2486-2501.

Grafton ST, Arbib MA, Fadiga L, Rizzolatti G (1996) Localization of grasp representations in humans by positron emission tomography. II. Observation compared with imagination. Exp Brain Res 112:103-111.

Kawashima R, Naitoh E, Matsumura M, Itoh H, Ono S, Satoh K, Gotoh R, Koyama M, Inoue K, Yoshioka S, Fukuda H (1996) Topographic representation in human intraparietal sulcus of reaching and saccade. NeuroReport 17:1253-1256.

Kertzman C, Schwarz U, Zeffiro TA, Hallett M (1997) The role of posterior parietal cortex in visually guided reaching movements in humans. Exp Brain Res 114:170-183.

Milner AD, Goodale MA (1995) The visual brain in action. New York: Oxford UP.

Mountcastle VB, Lynch JC, Georgopoulos A, Sakata H, Acuna C (1975) Posterior parietal association cortex of the monkey: command functions for operations within extrapersonal space. J Neurophysiol 38:871-908.

Murata A, Gallese V, Kaseda M, Sakata H (1996) Parietal neurons related to memory-guided hand manipulation. J Neurophysiol 75:2180-2186.

Mushiake H, Tanatsugu Y, Tanji J (1997) Neuronal activity in the ventral part of premotor cortex during target-reach movement is modulated by direction of gaze. J Neurophysiol 78:567-571.

Nakamura K, Chung HH, Graziano MS, Gross CG (1999) Dynamic representation of eye position in the parieto-occipital sulcus. J Neurophysiol 81:2374-2385.

Oldfield RC (1971) The assessment and analysis of handedness: the Edinburgh inventory. Neuropsychology 9:97-112.

Sakata H, Taira M, Murata A, Mine S (1995) Neural mechanisms of visual guidance of hand action in the parietal cortex of the monkey. Cereb Cortex 5:429-438.

Sakata H, Taira M, Kusunoki M, Murata A, Tanaka Y (1997) The Trends in Neuroscience lecture. The parietal association cortex in depth perception and visual control of hand action. Trends Neurosci 20:350-357.

Snyder LH, Batista AP, Andersen RA (1997) Coding of intention in the posterior parietal cortex. Nature 386:167-170.

Snyder LH, Grieve KL, Brotchie P, Andersen RA (1998) Separate bodyand world-referenced representations of visual space in parietal cortex. Nature 394:887-891.

Strupp JP (1996) Stimulate: a GUI based fMRI analysis software package. NeuroImage 3:S607.

Talairach J, Tournoux P (1988) Co-planar stereotaxic atlas of the human brain: 3-dimensional proportional system: an approach to cerebral imaging. Stuttgart, Germany: Thieme.

Trotter Y, Celebrini S (1999) Gaze direction controls response gain in primary visual-cortex neurons. Nature 398:239-242.

Vallar G (1998) Spatial hemineglect in humans. Trends Cognit Sci 2:87-97.

Zipser D, Andersen RA (1988) A back-propagation programmed network that simulates response properties of a subset of posterior parietal neurons. Nature 331:679-684. 